\title{
On a theorem of Manning-Cameron*
}

\author{
By Shiro Iwasaki
}

(Received December 25, 1975)

In 1929, Manning ([6]) proved that if $G$ is a uniprimitive permutation group on $\Omega$ (i.e., $(G, \Omega)$ is primitive, but not doubly transitive), and if the stabilizer $G_{a}$ of a point $a \in \Omega$ acts doubly transitively on an orbit of length $k>2$, then $G_{a}$ has an orbit whose length is greater than $k$ and a divisor of $k(k-1)$. Recently this was reproved more explicitly and strongly by Cameron ([1], [2]). In this short note, we remark that a similar result holds even when $G_{a}$ does not act doubly transitively on an orbit of length $k$.

Definitions and Notation. All permutation groups and sets considered in this note are finite. For definitions and notation, we follow those of Wielandt [7] and Higman [5]. Let $G$ be a transitive permutation group on a finite set $\Omega$. For $a \in \Omega, g \in G$ and a subgroup $H$ of $G$, we denote by $a^{g}$ the image of $a$ under $g$ and set $a^{H}=\left\{a^{g} \mid g \in H\right\}$. For a subset $S$ of $\Omega$, we set $S^{g}=\left\{a^{g} \mid a \in S\right\}, G_{S}=\left\{g \in G \mid a^{g}=a\right.$ for all $\left.a \in S\right\}$, and $G_{(S)}=\left\{g \in G \mid S^{g}=S\right\}$. If $S=\{a, b, \cdots\}, G_{S}$ is written $G_{a b \ldots}$.

The number of $G_{a}$-orbits on $\Omega$ counting the trivial orbit $\{a\}$ is independent of the choice of $a \in \Omega$ and is called the rank of $G$. If $(G, \Omega)$ is primitive and has rank greater than 2 , it is said uniprimitive. The lengths of the $G_{a^{-}}$orbits are called the subdegrees of $G$. Any $G_{a}$-orbit $\Delta(a)$ is chosen so that $\Delta(a)^{g}=\Delta\left(a^{g}\right)$ for all $a \in \Omega$ and all $g \in G$, and $\Delta$ is called an orbital of $G$. Each $\Delta(a)$ has a paired orbit defined by $\left\{a^{g^{-1}} \mid g \in G, a^{g} \in \Delta(a)\right\}$, which is also $G_{a}$-orbit and denoted by $\Delta^{\prime}(a) .|\Delta(a)|=\left|\Delta^{\prime}(a)\right|, \Delta^{\prime \prime}(a)=\Delta(a)$ by [7, $\left.\S 16\right]$, and

$$
b \in \Delta(a) \text { if and only if } a \in \Delta^{\prime}(b) .
$$

If $\Delta^{\prime}(a)=\Delta(a), \Delta$ or $\Delta(a)$ is said self-paired. Following Cameron [1], for orbitals $\Delta$ and $\Gamma$, we define

$$
(\Delta \circ \Gamma)(a)=\left\{b \in \Omega \mid \Delta(a) \cap \Gamma^{\prime}(b) \neq \phi, b \neq a\right\},
$$

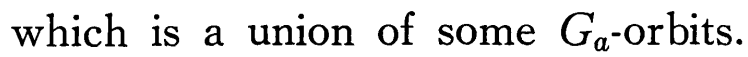

THEOREM. Let $G$ be a uniprimitive permutation group on a finite set

* This partly overlaps with "Jikken-Haichi no Kumiawase-Sugaku to Gunron", Res. Inst. Math. Sci., 1974, 75-82 (in Japanese). 
$\Omega$, and for $a \in \Omega$ let $\Delta(a)$ a $G_{a}$-orbit of length $k \geqq 2$ on which $G_{a}$ acts as rank $r$ group with subdegrees $1, k_{1}, \cdots, k_{r-1}\left(k=1+k_{1}+\cdots+k_{r-1}\right)$. Suppose, either
$(*)$
$\Delta$ (a) is self-paired, or
$(* *)$ $\left|G_{a}: G_{a \cup \Delta(a)}\right|$ is even.

Then, there exists a $G_{a}$-orbit $\Gamma(a)$ of length $l$ such that

(i) $\Gamma \neq \Delta, \Delta^{\prime}$ and $\Gamma(a) \subseteq\left(\Delta^{\prime} \circ \Delta\right)(a)$,

and for some $k_{i}(1 \leqq i \leqq r-1)$,

(ii) $k_{i}<l$ and $l$ is a divisor of $k k_{i}$,

(iii) if $b \in \Delta(a),|\Gamma(b) \cap \Delta(a)|=a$ sum of some $k_{j}$ 's containing $k_{i}$ (so $|\Delta(b) \cap \Delta(a)|$ is 0 or a sum of some $k_{j}$ 's, $\left.j \neq i\right)$.

Furthermore, if all the $r G_{a b}$-orbits on $\Delta(a)(b \in \Delta(a))$ are self-paired, $\Gamma(a)$ is self-paired.

Proof. Proof is almost trivial. Take a point $b \in \Delta(a)$. By assumption, $G_{a b}$ has $r$ orbits on $\Delta(a)$, say $\{b\}, \Delta_{1}, \cdots, \Delta_{r-1}$ with $\left|\Delta_{i}\right|=k_{i}$ (and so $\Delta(a)-\{b\}$ $\left.=\bigcup_{i=1}^{r-1} \Delta_{i}\right)$. First, we show that $\Delta(b) \nsupseteq \Delta(a)-\{b\}$ in the case $\left(^{*}\right)$ and that $\Delta(b) \cup \Delta^{\prime}(b) \nsupseteq \Delta(a)-\{b\}$ in case $\Delta$ is not self-paired and (**) holds. In the former case, if $\Delta(b) \supseteq \Delta(a)-\{b\}$, then $\{a\} \cup \Delta(a)=\{b\} \cup \Delta(b)$. This implies that, if we take $g \in G$ with $a^{g}=b$, then $g \in G_{(a \cup \Delta(a))}$ and so $G_{a} \lesseqgtr G_{(a \cup \Delta(a))} \lesseqgtr G$, which contradicts the primitivity of $(G, \Omega)$. In the latter case, suppose $\left(\Delta(b) \cup \Delta^{\prime}(b)\right)$ $\cap \Delta(a)=\Delta(a)-\{b\}$. By Higman [5, (4.2)], $|\Delta(b) \cap \Delta(a)|=\left|\Delta^{\prime}(b) \cap \Delta(a)\right|=(k-1) / 2$. Since $\Delta(b) \cap \Delta(a)$ is a union of some $G_{a b}$-orbits, we may set $\Delta(b) \cap \Delta(a)=\bigcup_{i=1}^{t} \Delta_{i}$. Then we have $\Delta^{\prime}(b) \cap \Delta(a)=\bigcup_{i=1}^{t} \Delta_{i}^{\prime}$, where $\Delta_{i}^{\prime}=\left\{b^{h^{-1}} \mid h \in G_{a}, b^{h} \in \Delta_{i}\right\}$, the paired orbit of $\Delta_{i}$, because for all $i, 1 \leqq i \leqq t, \Delta_{i}^{\prime}$ is contained in $\Delta^{\prime}(b)$ and $\Delta(a)$ by definition, $\Delta_{i} \neq \Delta_{j}$ implies $\Delta_{i}^{\prime} \neq \Delta_{j}^{\prime}$, and $|\Delta(b) \cap \Delta(a)|=\left|\Delta^{\prime}(b) \cap \Delta(a)\right|$. Thus $\Delta(a)=\{b\} \cup\left(\Delta_{1} \cup \Delta_{1}^{\prime}\right) \cup \ldots \cup\left(\Delta_{t} \cup \Delta_{t}^{\prime}\right)$ and the transitive permutation group $\left(G_{a} \mid\right.$ $\left.G_{a \cup \Delta(a)}, \Delta(a)\right)$ has no nontrivial self-paired orbit and so $\left|G_{a} / G_{a \cup \Delta(a)}\right|$ is odd by Wielandt [7, Theorem 16.5]. This contradicts the assumption (**). Therefore, in both cases there exists an element $c$ of some $\Delta_{i}$ such that $c \notin \Delta(b) \cup \Delta^{\prime}(b)$. Let $\Gamma(b)$ be a $G_{b}$-orbit containing $c$ and set $l=|\Gamma(a)|$. Then $\Gamma \neq \Delta, \Delta^{\prime}$. By definition $\left(\Delta^{\prime} \circ \Delta\right)(b) \ni c$ and so $\left(\Delta^{\prime} \circ \Delta\right)(b) \supseteq c^{G_{b}}=\Gamma(b)$, proving (i). $\Gamma(b) \cap \Delta(a)$ contains $c$ and so is a union of some $G_{a b}$-orbits containing $c^{G_{a b}}=\Delta_{i}$, proving (iii). Since $\left|G_{a}: G_{a b c}\right|=\left|G_{a}: G_{a b}\right| \cdot\left|G_{a b}: G_{a b c}\right|=k k_{i}$ and $\mid G_{b}$ : $G_{a b c}|=| G_{b}: G_{b c}|\cdot| G_{b c}: G_{a b c}|=l| G_{b c}: G_{a b c} \mid$, it follows that $l$ is a devisor of $k k_{i}$. 
$\Gamma(b) \cap \Delta(a) \supseteq \Delta_{i}$ implies $l \geqq k_{i}$. If $l=k_{i}$, then $k=\left|G_{b c}: G_{a b c}\right|=\left|a^{G_{b c}}\right|$. Since $G_{b c}$ acts on $\Delta^{\prime}(b) \cap \Delta^{\prime}(c)$ containing $a$, we have $\Delta^{\prime}(b) \cap \Delta^{\prime}(c) \supseteq a^{\theta_{b c}}$ and so $\Delta^{\prime}(b)=\Delta^{\prime}(c)$, which implies $G_{b} \lesseqgtr\left(\Delta^{\prime}(b)\right) \varsubsetneqq G$ and contradicts the primitivity of $(G, \Omega)$. Thus we have $l>k_{i}$, proving (ii).

Next we assume that, for $b \in \Delta(a)$, all the $G_{a b}$-orbits on $\Delta(a)$ are selfpaired. Since $\Gamma(b) \cap \Delta(a)$ is nonempty and a union of some $G_{a b}$-orbits on $\Delta(a)$, we may set $\Gamma(b) \cap \Delta(a)=\bigcup_{j=1}^{s} \Delta_{j}$. Then we have $\Gamma^{\prime}(b) \cap \Delta(a)=\bigcup_{j=1}^{s} \Delta_{j}^{\prime}$ as before. On the other hand, by assumption $\Delta_{j}^{\prime}=\Delta_{j}, 1 \leqq j \leqq s$ and so $\Gamma^{\prime}(b) \cap$ $\Delta(a)=\Gamma(b) \cap \Delta(a)$. Thus $\Gamma^{\prime}(b) \cap \Gamma(b)$ is nonempty and $\Gamma^{\prime}(b)=\Gamma(b)$. This completes the proof.

REMARK 1. If $r=2$ and $k>2$, Cameron [2] asserts $2 k \leqq l$ (and so $2(k-1) \leqq l)$. However, in general $2 k_{i} \leqq l$. For example, let $G$ be the Higman-

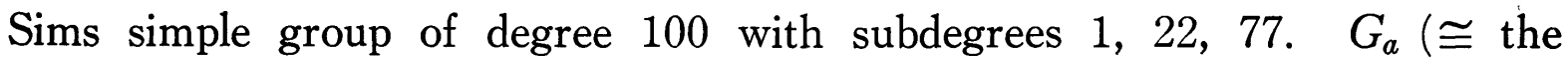
Mathieu group $M_{22}$ ) acts on the orbit of length 77 (the blocks of the associated Steiner system) as rank 3 group with subdgrees 1, 16, 60. Although $16<$ $22 \mid 77 \cdot 16,2 \cdot 16 \npreceq 22$.

From (ii) and the last assertion of Theorem, we have immediately

Corollary 1. Let $G$ be a uniprimitive permutation group on $\Omega$, and for $a \in \Omega \quad \Delta(a)$ a $G_{a}$-orbit with $|\Delta(a)| \geqq 2$. Suppose $|\Delta(a)|<|\Gamma(a)|$ for any $G_{a}$-orbit $\Gamma(a)$ different from $\{a\}$ and $\Delta(a)$. Then $G_{a}$ does not act regularly on $\Delta(a)$.

CoROllaRy 2. There exists no uniprimitive permutation group $(G, \Omega)$ such that $G_{a}(a \in \Omega)$ has only one nontrivial self-paired orbit $\Delta(a)$ and for $b \in \Delta(a)$, all the $G_{a b}$-orbits on $\Delta(a)$ are self-paired.

REMARK 2. The simple unitary group $P S U\left(3,3^{2}\right)$ has a representation as a primitive group $G$ of rank 4 such that $G_{a} \cong P S L(3,2)$ and the subdegrees are $1,21,7,7$, and the $G_{a}$-orbits of lenth 7 are paired. However, $G_{a}$ on the $G_{a}$-orbit of length 21 has subdegrees $1,2,2,4,4,8$ and the orbits of length 4 are paired.

Incidentally we add

Proposition. Let $(G, \Omega)$ be a transitive permutation group and for $a \in \Omega$ let $\Delta(a)$ and $\Gamma(a)$ be $G_{a}$-orbits different from $\{a\}$. Let $m$ be the number of $G_{a}$-orbits contained in $\left(\Delta^{\prime} \circ \Gamma\right)(a)$ and for some $b \in \Delta(a)$ let $t$ be the number of $G_{a b}$-orbits on $\Gamma(a)$. Then we have

(i) $1 \leqq m \leqq t-1$ if $\Gamma=\Delta$ (i.e., $G_{a}$ acts on $\Delta(a)$ as a group of rank $t$ ) and $|\Gamma(a)| \geqq 2$. In particular, if $G_{a}$ acts doubly transitively on $\Delta(a)$, then $\left(\Delta^{\prime} \circ \Delta\right)(a)$ is a self-paired $G_{a}$-orbit. 
(ii) $1 \leqq m \leqq t$ if $\Gamma \neq \Delta$. In particular, if $|\Delta(a)|$ and $|\Gamma(a)|$ are relatively prime and $|\Delta(a)|$ or $|\Gamma(a)| \geqq 2$, then $(\Delta \circ \Gamma)(a)$ is a single $G_{a}$-orbit.

Proof. Let $\Gamma_{0}(a)=\{a\}, \Gamma_{1}(a)=\Delta(a), \Gamma_{2}(a)=\Gamma(a), \Gamma_{3}(a), \cdots$ be the set of $G_{a}$-orbits on $\Omega$. Following Higman [5] we set $\mu_{i j}^{(\alpha)}=\left|\Gamma_{\alpha}(c) \cap \Gamma_{i}(a)\right|$ for $c \in \Gamma_{j}(a)$. Then, by definition $\left(\Delta^{\prime} \circ \Gamma\right)(a)=\underset{\substack{\left(a^{\prime}\right) \\ i \neq 0, u^{\prime}, \neq}}{\bigcup} \Gamma_{i}(a)$, where $\Gamma_{j^{\prime}}(a)=\Gamma_{j}^{\prime}(a)$. By [5, (4.2)], $\mu_{1^{\prime} i}^{\left(2^{\prime}\right)} \neq 0$ if and only if $\mu_{i 1^{\prime}}^{(2)} \neq 0$, and so $\left(\Delta^{\prime} \circ \Gamma\right)(a)=\underset{\substack{(2) \\ i \neq 0, \mu_{i 1^{\prime} \neq 0}}}{\bigcup} \Gamma_{i}(a)$. Set $\left(\Delta^{\prime} \circ \Gamma\right)(a)=$ $\bigcup_{j=1}^{m} \Gamma_{i_{j}}(a)$. Then, for all $j, 1 \leqq j \leqq m, \Gamma(a) \cap \Gamma_{i_{j}}(b)$ is nonempty and a union of some $G_{a b}$-orbits on $\Gamma(a)$. Therefor we have $m \leqq\left(\right.$ the number of $G_{a b}$-orbits on $\Gamma(a))=t$. In particular, if $\Gamma=\Delta$, then $b \in \Gamma(a)$ and $m \leqq($ the number of $G_{a b}$-orbits on $\Gamma(a)$ different from $\left.\{b\}\right)=t-1$. Since $\sum_{i} \mu_{i 1^{\prime}}^{(2)}=|\Gamma(a)|$ and $\Gamma_{00^{\prime}}^{(2)}=1$ or 0 according as $\Gamma=\Delta$ or $\Gamma \neq \Delta, \mu_{i 1^{\prime}}^{(0)} \neq 0$ for some $i \neq 0$ (in case $\Gamma=\Delta$, $|\Gamma(a)| \geqq 2$ by assumption) and so $m \geqq 1$. In general, since $\mu_{1^{\prime} i}^{\left(1^{\prime}\right)}=\mu_{1^{\prime} i^{\prime}}^{\left(1^{\prime}\right)}$, if $\left(\Delta^{\prime} \circ \Delta\right)(a)$ contains a $G_{a}$-orbit $\Gamma_{i}(a)$, then it does also the paired orbit $\Gamma_{i}^{\prime}(a)$, so the 'in particular' part of (i) is obvious. If $|\Delta(a)|$ and $|\Gamma(a)|$ are relatively prime and $|\Delta(a)|$ or $|\Gamma(a)| \geqq 2$, then $\Delta \neq \Gamma$ and by [7, theorem 17.3] $G_{a b}$ is transitive on $\Gamma(a)$, i.e., $t=1$, hence $m=1$, i. e., $\left(\Delta^{\prime} \circ \Gamma\right)(a)$ is a $G_{a}$-orbit. Therefore, by replacing $\Delta^{\prime}$ by $\Delta,(\Delta \circ \Gamma)(a)$ is a $G_{a}$-orbit.

Theorem is a little available in dealing with primitive extensions of rank 3 of some permutation groups. Here we say a permutation group $(G, \Omega)$ is a primitive extension of rank 3 of a transitive permutation group $(H, \Delta)$ if $(G, \Omega)$ is primitive and has rank 3 and there exists an orbit $\Delta(a)$ of a stabilizer $G_{a}, a \in \Omega$, such that $G_{a}$ is faithful on $\Delta(a)$ and $\left(G_{a}, \Delta(a)\right)$ is isomorphic to $(H, \Delta)$. For example, the following simple groups have no primitive extensions of rank 3 (Here, for a group $H$ and its subgroup $K$, " $H>K$ " denotes the representation of $H$ on $K$ ).

1) $\operatorname{PSU}\left(3,5^{2}\right)>A_{7}$

2) The Janko's simple group of order $175560>P S L(2,11)$

3 ) Mclaughlin's simple group $>P S U\left(4,3^{2}\right)$

4) Higman-Sims simple group $>M_{22}$

Indeed, assume that each one of the above groups has a primitive rank 3 extension $(G, \Omega)$ and let $\{a\}, \Delta(a), \Gamma(a)$ be the $G_{a}$-orbits with $|\Delta(a)|=k$, $|\Gamma(a)|=l$, and set $\lambda=|\Delta(a) \cap \Delta(b)|$ for $b \in \Delta(a)$. As to 1), by Theorem, $7<l \mid 50 \cdot 7$ with $\lambda=0$ or 42 , or $42<l \mid 50 \cdot 42$ with $\lambda=0$ or 7 . However, by Higman [4, Lemma 7] we have $l=30,45$ or 48 if $l<50$, and $l=50 \cdot 49 / 2$ if $l \geqq 50$ and $\lambda=0,7$ or 42 . These are inconsistent. As to 3$)$, take $c \in \Gamma(a)$. $G_{a c}$ is contained in a maximal subgroup of $G_{a}$ and by Finkelstein [3, Theorem 
1] we have $l \geqq k=275$. By [4, Lemma 7] and Theorem, the case $l=330$, $\lambda=112$ remains. However, by [3] $G_{a}$ has no maximal subgroup whose index is a divisor of 330 . Likewise, in another cases we have a contradiction. Here we use a table of parameters of possible rank 3 permutation groups made by Dr. H. Enomoto on the basis of [4, Lemma 7] with the help of a computer, and the author thanks him.

\section{References}

[1] P. J. CAmeron: Proofs of some theorems of W. A. Manning, Bull. London Math. Soc. 1 (1969), 349-352.

[2] P. J. CAMERon: Permutation groups with multiply transitive suborbits, Proc. London Math. Soc. (3), 25 (1972), 427-440.

[3] L. FinkELSTEIN : The maximal subgroups of Conway's group $\mathrm{C}_{3}$ and McLaughlin's group, J. Alg. 25 (1973), 58-89.

[4] D. G. HigmaN : Finite permutation groups of rank 3, Math. Z. 86 (1964),145-156.

[5] D. G. HigmAN: Intersection matrices for finite permutation groups, J. Alg. 6 (1967), 22-42.

[6] W. A. MANNING: A theorem concerning simply transitive primitive groups, Bull. Amer. Math. Soc. 35 (1929), 330-332.

[7] H. Wielandt: Finite permutation groups, Academic Press, New York and London, 1964.

Mathematics, Faculty of commerce, Hitotsubashi University
Department of Mathematics, Hokkaido University 